\title{
Range extension of the inter-vehicle communication via the spatial diversity
}

\author{
Chi-Min Li ${ }^{1}$, Pao-Jen Wang ${ }^{2 a)}$, and Cheng-Ying $\mathbf{W u}^{1}$ \\ ${ }^{1}$ Department of Communications, Navigation and Control Engineering, National \\ Taiwan Ocean University \\ 2 Pei-Ning Road, Keelung, 20224, Taiwan \\ ${ }^{2}$ Department of Electrical Engineering, Ming Chi University Of Technology \\ 84 Gungjuan Road., Taishan, Taipei 24301, Taiwan \\ a)pjwang@mail.mcut.edu.tw
}

\begin{abstract}
Currently, Intelligent Transportation System (ITS) is a promising technique that can efficiently manage the serious traffic problems in metropolises. It adopts the mobile communication techniques to transmit traffic information in many applications such as the inter-vehicle communications (IVC) and the vehicle-road communications (VRC). The longer distance of the information is transmitted, the more safety can be achieved for the drivers because they will have enough time to avoid or reduce the damage. In this paper, combined with a multi-ray shadowing model, the range extension capability by using the two receiving antennas is evaluated.
\end{abstract}

Keywords: ITS, IVC, shadowing model, spatial diversity

Classification: Wireless communication hardware

\section{References}

[1] S. Tsugawa, "Issues and Recent Trends in Vehicle Safety Communications," IATSS RESEARCH, vol. 29, no. 1, pp. 7-15, 2005.

[2] D. Jiang, V. Taliwal, A. Meier, W. Holfelder, and R. Herrtwich, "Design of $5.9 \mathrm{GHz}$ DSRC-Based Vehicular Safety Communication," IEEE Trans. Wireless Commun., vol. 13, pp. 36-43, Oct. 2006.

[3] P. J. Wang, C. M. Li, H. J. Li, and C. Y. Wu, "A Channel Awareness Vehicle Detector," IEEE Trans. Intell. Transport. Syst., vol. 11, no. 2, pp. 339-347, June 2010.

[4] S. Kato and S. Tsugawa, "Evaluation of Information Transmission over Inter-Vehicle Communication with Simulation Studies," IEEE 5th Int. Conf. Intell. Transport. Syst., pp. 324-329, Sept. 2002.

[5] P. J. Wang, C. M. Li, and H. J. Li, "Influence of the Shadowing to the Information Transmission Distance in Inter-Vehicle Communications," 20th IEEE Int. Symp. Personal, Indoor and Mobile Radio Communications (PIMRC), Japan, Sept. 2009.

[6] T. S. Rappaport, Wireless Communications: Principles and Practice, 2nd ed., New Jersey: Prentice Hall, 2002.

[7] I. Sen and D. W. Matolak, "Vehicle-Vehicle Channel Models for the 5-GHz Band," IEEE Trans. Intell. Transport. Syst., vol. 9, no. 2, pp. 235-245, June 2008. 


\section{Introduction}

Intelligent Transportation System (ITS) is a smart system that integrates communication, information, electronics technologies, and traffic management to efficiently manipulate public transportation. It adopts the mobile communication techniques to transmit traffic information in many applications such as the inter-vehicle communications (IVC) and the vehicle-road communications (VRC). In the IVC, if the vehicle has a high risk of collision, an emergency signal will be initiated from a forerunning vehicle to reduce the property loss and personal injuries $[1,2,3]$. Basically, the longer distance of the emergency information is transmitted, the more safety can be achieved for the drivers because they will have enough time to avoid or reduce the damage. However, the ability of effective transmission distance of the vehicular information depends notably on the percentage of the vehicles equipped with IVC communication device and the characteristics of radio wave propagation [4]. Furthermore, those vehicles which are not equipped with the communication devices will block the vehicle information communication and result in the undesirable shadowing attenuation.

Besides, it is well-known that the adoption of multiple antennas at the receiving end is a very effective method to increase the received signal to noise ratio (SNR) and has the advantage of the spatial diversity. Combined with multi-ray shadowing model [5], in this paper, we evaluate the improvement of the effective transmission distance or the range extension by using two receiving antennas for the IVC scenario. This paper is organized as follows: In Section 2, the adopted IVC shadowing model is briefly reviewed. Section 3 conducts the computer simulations to illustrate the improvement of the effective transmission distance for the IVC system via using two different receiving antennas. Finally, some conclusions of this paper are given in Section 4.

\section{Descriptions of the shadowing method}

To model the shadowing effect in IVC, we use a multi-ray shadowing model proposed in [5] (Fig. 1). For the conventional knife-edge and the Bullington's methods [6], both consider only the wave propagation through the top of the shield object. In the scenario of the IVC, these methods are not suitable because the vehicles affect the shadowing degradation only on the blocking portion of the propagation from the transmitter (Tx). Assume if there are shield vehicles between the $\mathrm{Tx}$ and the receiver $(\mathrm{Rx}), \mathrm{Rx}$ receives the signal coming mainly from four propagation paths, i.e., one is diffracted from the top of the vehicle, one is from the bottom of the vehicle, and the other two are diffracted at the both door-edges of the vehicle. It assumes that each path suffers the attenuation which can be estimated from the conventional knife-edge method or the equivalent obstacle of the Bullington's method.

The procedures of the multi-ray shadowing model are summarized as:

Step 1: Use the knife-edge method or the Bullington's method to represent the shield objects as a single equivalent obstacle. 
Step 2: Calculate the four effective heights: one is the distance from the top of the shield object to the link of the Tx and Rx, one is from the link to the bottom of the shield object while the other two effective heights are determined from the both door-edges of the vehicle.

Step 3: Use the knife-edge method for these paths to predict the resulted electric fields.

Step 4: Sum up these electric fields to calculate the path loss.

\section{Computer simulations}

In the simulations, we simulate the relation of the transmission distance versus the penetration rate and verify the range extension capability via the spatial diversity technique [4]. The penetration rate is defined as the percentage of the vehicles equipped with IVC communication device. The shadowing effect of the vehicles without the IVC communication devices is evaluated by the multi-ray shadowing model. Each vehicle contains one transmitting and two receiving antennas. Channel attenuations for the two receiving antennas are assumed to be independent to have the full diversity advantage.

Two scenarios are conducted: one is the Line-of-Sight (LOS) case and the other is Non-LOS (NLOS) case. During the signal transmission, assume all vehicles have the same speed. Besides, vehicles with the IVC communication device transfer the traffic information only in the backward direction. If there is no shielded vehicle between the $\mathrm{Rx}$ and the $\mathrm{Tx}$, the transmission channel is modeled as a LOS scenario and the instantaneous received power is calculated via the Friss equation with the Rician fading. Otherwise, the transmission channel is modeled as a NLOS scenario and the instantaneous received power is calculated by the Friss equation with the Rayleigh fading and the shadowing effect $[6,7]$.

We use a time headways distribution provided in [4] to generate the distance between two successive vehicles. Multiplying the speed with the random generated time headway, the distance between two successive vehicles can be obtained.

The penetration rate can be used to determine whether or not the successive vehicle was equipped with the IVC communication device. If not, the vehicle will have the shadowing effect for the signal transmission. The transmitted power of the signal is $10 \mathrm{dBm}$ and the sensitivity of the received power is $-85 \mathrm{dBm}$. That is, if the received power is higher than the $-85 \mathrm{dBm}$, we accumulate the distance and the $\mathrm{Rx}$ of vehicle emits an equal power signal $(10 \mathrm{dBm})$ in the backward direction. The calculation of the transmission distance will be terminated if the received signal power is below the sensitivity $(-85 \mathrm{dBm})$ and the effective transmission distance is equal to the accumulated distance from the vehicle initiating the signal.

Fig. $2 \sim 3$ depict the effective transmission distance versus the penetration rate for different speeds of the vehicles. Obviously, the effective transmission distance will be increased as the penetration rate increases. Besides, the low- 


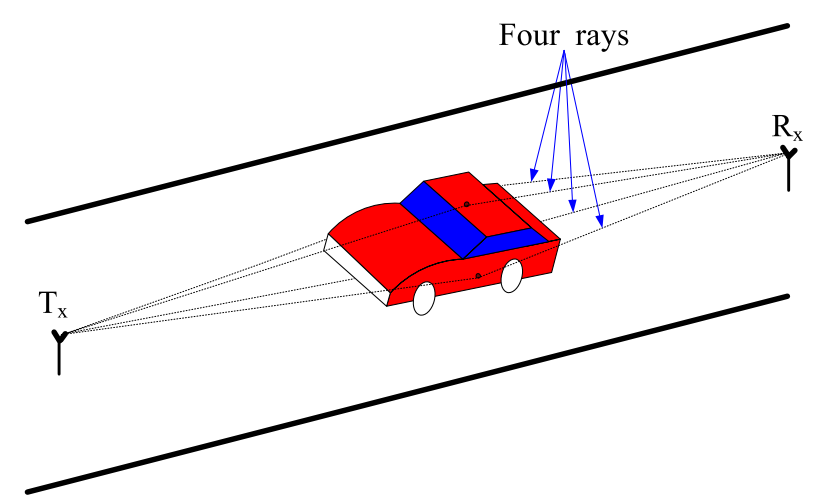

Fig. 1. Multi-ray Shadowing Model of the IVC.

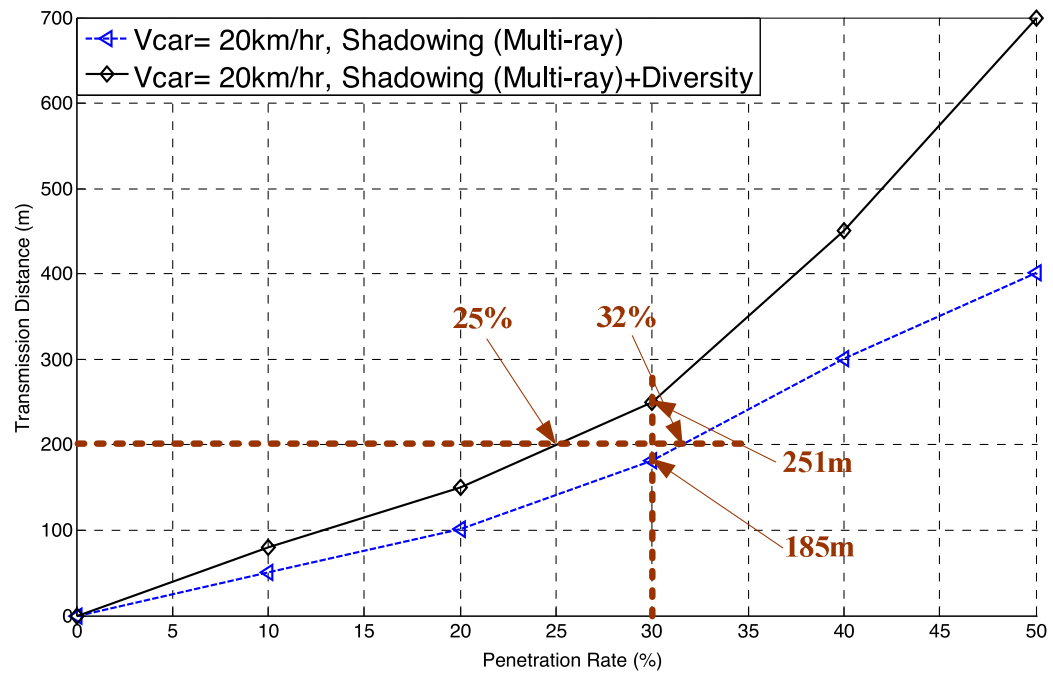

Fig. 2. Relations Between Transmission Distance versus Penetration Rate at Speed $20 \mathrm{~km} / \mathrm{h}$.

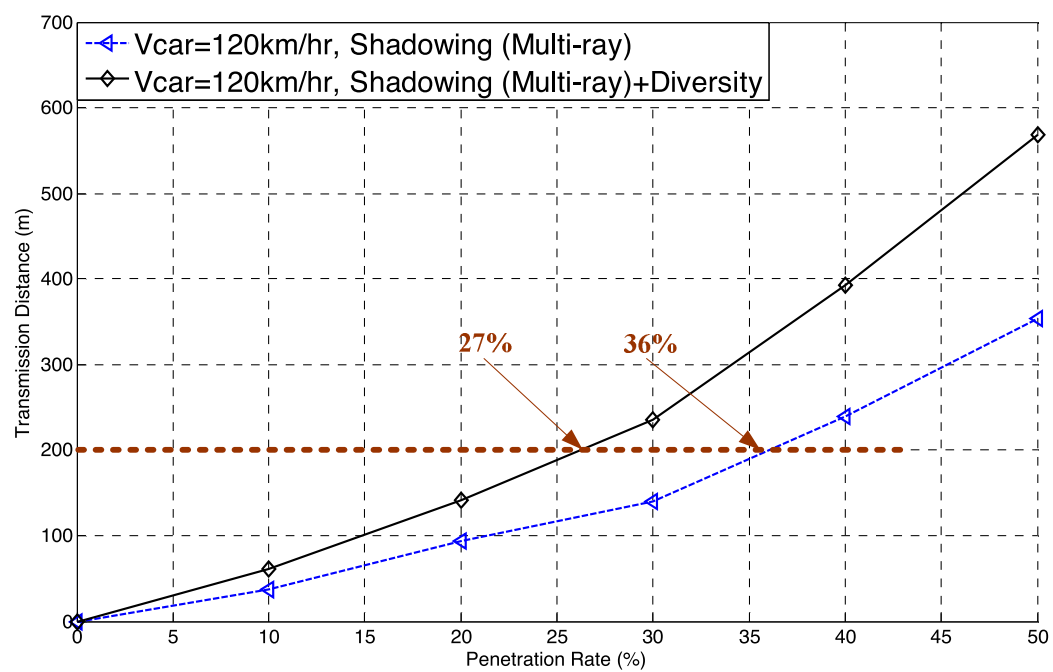

Fig. 3. Relations Between Transmission Distance versus Penetration Rate at Speed $120 \mathrm{~km} / \mathrm{h}$. 
speed vehicles have longer transmission distance than the high-speed vehicles at the same penetration rate. This comes from the fact that the distance between two successive vehicles for the low-speed case is smaller than the high-speed case under the same time headway condition. Therefore, the lowspeed IVC signals will suffer less attenuation and have longer transmission distance than the high-speed case.

Fig. $2 \sim 3$ also illustrate the range extension capabilities by using the two receiving antennas. Both reveal that the spatial diversity can greatly increase the effective transmission distance. For example, in the $20 \mathrm{~km} / \mathrm{h}$ case, the distance can be increase from $185 \mathrm{~m}$ to $251 \mathrm{~m}$ at the $30 \%$ penetration rate. Results also show that if the transmission distance for a safety purpose should be greater than $200 \mathrm{~m}$, the penetration rate can be reduced from $32 \%$ to $25 \%$ for the $20 \mathrm{~km} / \mathrm{h}$ case. For the $120 \mathrm{~km} / \mathrm{h}$ case, it can reduce the penetration rate from $36 \%$ to $27 \%$.

\section{Conclusions}

This paper surveys the range extension capability via using the spatial diversity technique for the IVC system. Based on the simulated results, the transmission distance can be greatly increased with the help of spatial diversity. Besides, for a safety distance requirement, the spatial diversity technique can also reduce the penetration rate for the IVC system that make the IVC communication more practical in the future related applications.

\section{Acknowledgments}

This research work was supported by the National Science Council of the Republic of China under the Grant number NSC 99-2221-E-019-023-. 